\title{
Estudo de caso-controle em jovens moradores de regiões fluoretadas e não
}

\section{fluoretadas}

\author{
Case-control study in young people living in fluoridated and non-fluoridated regions \\ Estudio de casos y controles en jóvenes que viven en regiones fluoradas y no fluoradas
}

Recebido: 30/04/2021 | Revisado: 06/05/2021 | Aceito: 07/05/2021 | Publicado: 22/05/2021

Suzely Adas Saliba Moimaz

ORCID: https://orcid.org/0000-0002-4949-529X Universidade Estadual Paulista, Brasil

E-mail: suzely.moimaz@unesp.br

Luís Felipe Pupim dos Santos

ORCID: https://orcid.org/0000-0003-2110-7360 Universidade Estadual Paulista, Brasil E-mail:1fpupim@hotmail.com

Tânia Adas Saliba

ORCID: https://orcid.org/0000-0003-1327-2913 Universidade Estadual Paulista, Brasil E-mail: tania.saliba@unesp.br

Nemre Adas Saliba

ORCID: https://orcid.org/0000-0001-9608-1631 Universidade Estadual Paulista, Brasil E-mail: nemre.saliba@unesp.br

Fernando Yamamoto Chiba

ORCID: https://orcid.org/0000-0003-4406-405X Universidade Estadual Paulista, Brasil

E-mail: fernando.chiba@unesp.br

Orlando Saliba

ORCID: https://orcid.org/0000-0003-1439-4197 Universidade Estadual Paulista, Brasil E-mail: osaliba@terra.com.br

\begin{abstract}
Resumo
Objetivou-se verificar a prevalência e a severidade da cárie dentária em jovens de 12 anos de um município que realiza regularmente a fluoretação das águas de abastecimento público, desde 1972, comparando as condições de saúde bucal entre jovens nascidos e não nascidos no local. Trata-se de um estudo de caso-controle, realizado em 164 escolares de 12 anos matriculados em escolas públicas de um município que realiza ininterruptamente a fluoretação das águas do sistema público de abastecimento. $\mathrm{O}$ grupo controle foi composto por jovens com $\mathrm{CPOD}=0(\mathrm{n}=82)$ e o grupo caso foi composto por jovens com $\mathrm{CPOD}>0(\mathrm{n}=82)$. $\mathrm{O}$ fato de não ter nascido e vivido sempre no município foi considerado o fator de exposição. A avaliação da condição dentária foi realizada utilizando-se o índice CPOD. Verificou-se que o índice CPOD médio dos 164 escolares foi de $1,00 \pm 1,24$. Houve associação estatisticamente significante $(\mathrm{p}=0,001)$ entre a experiência de cárie dentária e o fator de exposição, apresentando odds ratio= 3,0134 (IC 95\%= 1,5944 - 5,6953). As médias do índice CPOD (não-expostos= $0,44 \pm 0,67$; expostos= $1,31 \pm 1,32$ ), do componente dentes cariados (nãoexpostos $=0,12 \pm 0,34$; expostos $=0,49 \pm 0,89$ ) e do componente dentes obturados (não-expostos $=0,40 \pm 0,73$; expostos $=$ $0,83 \pm 1,05)$ foram significativamente menores $(\mathrm{p}<0,05)$ nos adolescentes que nasceram e viveram sempre no município que realiza regularmente a fluoretação das águas de abastecimento público, em comparação aos nascidos em outro município. Conclui-se que houve associação entre o acesso à água fluoretada regularmente desde o nascimento e menor prevalência e severidade de cárie dentária.
\end{abstract}

Palavras-chave: Epidemiologia; Saúde bucal; Cárie dentária; Fluoretação.

\begin{abstract}
The objective of this study was to verify the prevalence and severity of dental caries in 12-year-olds from a municipality that regularly performs fluoridation of public water supply, since 1972, comparing oral health conditions among young people born and unborn at the site. This is a case-control study, carried out on 164 12-year-old schoolchildren enrolled in public schools in a municipality that continuously performs fluoridation of the water in the public water supply system. The control group was composed of young people with DMFT $=0(n=82)$ and the case group was composed of young people with DMFT $>0(n=82)$. The fact that he was not always born and lived in the municipality was considered the exposure factor. The evaluation of the dental condition was performed using the DMFT index. It was found that the average DMFT index of 164 students was $1.00 \pm 1.24$. There was a statistically significant association ( $\mathrm{p}=0.001)$ between
\end{abstract}


dental caries experience and the exposure factor, with odds ratio=3.0134 (95\% CI=1.5944 - 5.6595). The means of the DMFT index (unexposed $=0.44 \pm 0.67$; exposed $=1.31 \pm 1.32$ ), of the decayed teeth component (unexposed $=0.12 \pm 0.34$; exposed $=0.49 \pm 0.89$ ) and the filled teeth component (unexposed $=0.40 \pm 0.73$; exposed $=0.83 \pm 1.05$ ) were significantly lower $(\mathrm{p}<0.05)$ in the adolescents who were born and lived always in the municipality that regularly carries out the fluoridation of public water supply, compared to those born in another municipality. It was concluded that there was an association between access to fluoridated water regularly since birth and lower prevalence and severity of dental caries. Keywords: Epidemiology; Oral health; Dental caries; Fluoridation.

\section{Resumen}

El objetivo de este estudio fue verificar la prevalencia y severidad de la caries dental en niños de 12 años de un municipio que regularmente realiza la fluoración del suministro público de agua, desde 1972, comparando las condiciones de salud bucal entre jóvenes nacidos y no nacidos en el sitio. Se trata de estudio de casos y controles realizado en 164 escolares de 12 años matriculados en escuelas públicas de un municipio que continuamente realiza la fluoración del agua en el sistema público de abastecimiento. El grupo control estaba compuesto por jóvenes con CPOD=0 ( $\mathrm{n}=82)$ y el grupo casos estaba compuesto por jóvenes con CPOD $>0 \quad(n=82)$. Se consideró factor de exposición el hecho de que no siempre nació y vivió en el municipio. La evaluación de la condición dental se realizó mediante el índice CPOD. Se encontró

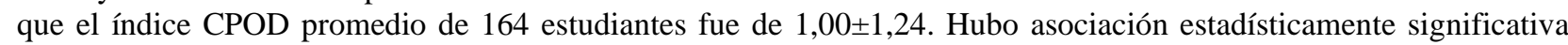
$(\mathrm{p}=0,001)$ entre la experiencia de caries dental y el factor de exposición, presentando odds ratio=3,0134 (IC 95\%=1,5944-5,6595). Las medias del índice CPOD (no expuestos $=0,44 \pm 0,67$; expuestos $=1,31 \pm 1,32$ ), del componente dientes cariados (no expuestos $=0,12 \pm 0,34$; expuestos $=0,49 \pm 0,89$ ) y del componente dientes obturados (no expuestos $=0,40 \pm 0,73$; expuestos $=0,83 \pm 1,05)$ fueron significativamente menores $(\mathrm{p}<0,05)$ en los adolescentes que nacieron y vivieron siempre en el municipio que regularmente realiza la fluoración del suministro público de agua, en comparación con los nacidos en otro municipio. Se concluyó que existía asociación entre el acceso regular al agua fluorada desde el nacimiento y una menor prevalencia y gravedad de la caries dental.

Palabras clave: Epidemiología; Salud bucal; Caries dental; Fluoración.

\section{Introdução}

Os problemas de saúde bucal de uma população podem ser determinados por meio da epidemiologia, que é a ciência que analisa os diversos fatores causadores da propagação de doenças e de determinados eventos que afetam a comunidade, por meio de índices e coeficientes que indicam a frequência. Os resultados de um inquérito epidemiológico odontológico são essenciais para os profissionais de saúde pública, pois orientam o planejamento, a execução e a avaliação das ações de saúde pública para a população (Frazão et al., 2021; Mota et al., 2020; Dye, Hsu, \& Afful, 2015).

A cárie dentária é considerada uma condição degenerativa, crônica e multifatorial que afeta um grande número de crianças e adolescentes em todo o mundo, podendo gerar dor, dificuldades de mastigação, problemas de fala, distúrbios gerais de saúde, problemas psicológicos e pior qualidade de vida (Silva et al., 2020; Carolino et al., 2020; Hall et al., 2017; Souza et al., 2017; Abanto et al., 2014). A condição socioeconômica de uma população pode ser considerada o principal fator que influencia a prevalência e a severidade da cárie dentária, a qual pode ser agravada por outros fatores, tais como a insuficiência de assistência odontológica e a falta de programas educativos e preventivos (Righolt et al., 2018; Garbin et al., 2017). Por outro lado, o controle do consumo de açúcar, implementação de ações de prevenção e promoção de saúde, melhoria da qualidade de vida e maior utilização do flúor por meio das águas de abastecimento público, dentifrícios e bochechos são medidas que podem contribuir para o declínio da prevalência da doença (Garbin et al., 2017; Baghlaf et al., 2018; Araújo et al., 2018).

Nas últimas décadas, tem ocorrido uma redução na prevalência da doença, com consequente aumento do número de indivíduos que nunca tiveram experiência de cárie dentária, entretanto, ainda é a principal doença bucal e um grave problema de saúde pública, e suas consequências, como a perda dentária, geram preocupação aos sistemas de saúde (Gomes \& Ros, 2008). De acordo com o Banco de Dados Global de Saúde Bucal da Organização Mundial da Saúde (OMS), a cárie dentária afeta cerca de 60 a 90\% das crianças em idade escolar e, em países de baixa e média renda, a incidência da doença tende a ser ainda maior (World Health Organization, 2015). Assim, torna-se necessário o monitoramento constante desta condição, principalmente nas populações de baixa renda e com menor possibilidade de acesso aos serviços de assistência e prevenção. Dentre os métodos preventivos de carie dentária, destaca-se a fluoretação das águas de abastecimento público, um método coletivo de uso do flúor, 
seguro, de grande abrangência e eficaz (Moimaz et al., 2017). Revisões sistemáticas têm demonstrado que localidades com água fluoretada apresentam maior número de crianças livres da doença em comparação com regiões não fluoretadas (McDonagh et al., 2000; Iheozor-Ejiofor et al., 2015).

As consequências da cárie dentária podem diminuir a qualidade de vida dos indivíduos afetados e desencadear outros agravos à saúde, justificando a continuidade dos programas de educação, promoção e prevenção da saúde bucal, bem como das ações de vigilância epidemiológica. O objetivo neste estudo foi verificar a prevalência e a severidade da cárie dentária em escolares de 12 anos de idade da rede pública de ensino de um município que adiciona flúor à rede pública de abastecimento de água regularmente, comparando as condições de saúde bucal entre nascidos e não nascidos no local.

\section{Metodologia}

Trata-se de um estudo observacional, analítico, de caso-controle, no qual foi investigada a prevalência e a severidade da cárie dentária em jovens de 12 anos da rede pública de ensino de um município do estado de São Paulo, no ano de 2019. A pesquisa foi conduzida de acordo com as recomendações das diretrizes do protocolo STROBE para estudos de caso-controle (Malta et al., 2010). O estudo foi realizado na cidade de Araçatuba, localizada na região noroeste do Estado de São Paulo, que possui uma área territorial de aproximadamente 1.167,126 km2, população de 198.129 habitantes, taxa de escolarização, para indivíduos de 6 a 14 anos, de 97,6\% e um Índice de Desenvolvimento Humano de 0,788 (Instituto Brasileiro de Geografia e Estatística, 2021). Destaca-se que o município realiza regularmente, desde 1972, o método da fluoretação das águas de abastecimento público.

Neste estudo, o grupo controle foi composto pelos escolares com índice $\mathrm{CPOD}=0$, ou seja, aqueles que nunca tiveram experiência de cárie dentária; e o grupo caso foi composto pelos escolares com CPOD>0, indicando que o indivíduo tem ou teve a doença; e não ter nascido e vivido sempre no município com fluoretação das águas foi considerado o fator de exposição para ocorrência da doença.

Foi realizado o mapeamento de todas as escolas da rede pública de ensino do município que apresentavam estudantes de 12 anos, sendo identificadas 22 escolas públicas e o total de 3265 escolares, com a referida idade, regularmente matriculados. Os critérios de inclusão considerados foram: presença de pelo menos 20 dentes na cavidade bucal; equilíbrio entre o número de voluntários do sexo masculino e feminino; estarem regularmente matriculados em escolas públicas do município; ausência de traumatismos faciais e ou outras condições que impossibilitassem o exame bucal. Foram excluídos os indivíduos com síndromes e má formações congênitas, periodontite avançada, deficiências psicológicas e mentais, aqueles que não assinaram o termo de assentimento livre e esclarecido (TALE) e/ou cujos responsáveis não assinaram o termo de consentimento livre e esclarecido (TCLE).

Previamente à coleta dos dados, foi realizado um estudo-piloto com 20 voluntários não incluídos na amostra do estudo, para verificar a necessidade de adequações no instrumento de coleta de dados e auxiliar o cálculo do tamanho da amostra. A partir dos dados do estudo-piloto, o tamanho da amostra do estudo foi calculado, com auxílio do software EpiInfo versão 7.2.4,

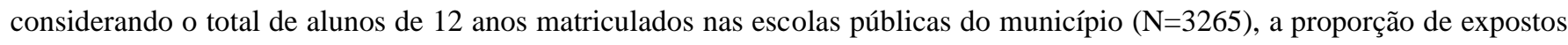
entre os indivíduos do grupo caso, a proporção de expostos entre os indivíduos do grupo controle, nível de confiança de 95\% e poder estatístico de $80 \%$, determinando-se o valor mínimo de 82 indivíduos para compor o estudo, sendo 41 para cada grupo. Considerando possíveis desistências e perda de segmento amostral, o tamanho amostral mínimo foi dobrado, de forma que a amostra foi composta por 164 escolares, sendo 82 para cada grupo. Inicialmente, realizou-se uma amostragem por conglomerados, a partir da qual foram selecionadas 14 escolas, de modo que instituições localizadas nas regiões central e periférica da cidade foram incluídas no estudo de forma proporcional e, posteriormente, foi realizada uma amostragem aleatória simples para a seleção dos escolares, respeitando-se o equilíbrio entre variáveis socioeconômicas. 
Para investigar a prevalência e a severidade da cárie dentária foi utilizado o índice CPOD, de acordo com os códigos e critérios recomendados pela Organização Mundial da Saúde, segundo o Manual para Levantamentos em Saúde Bucal (World Health Organization, 2013). Os exames foram realizados nos pátios das escolas, em local adequadamente ventilado e com luz natural, utilizando a sonda periodontal milimetrada da OMS e espelho bucal plano.

Os dados foram coletados por 2 equipes de examinadores e anotadores previamente treinados e calibrados. O processo de calibração consistiu na apropriação dos fundamentos teóricos das variáveis utilizadas; compreensão dos critérios a serem adotados para a definição de cada observação de exame e seus respectivos códigos; aplicação dos critérios em situações reais, ou seja, a própria calibração; e cálculo dos erros intra e interexaminadores, por meio do coeficiente Kappa. Por meio do cálculo do coeficiente Kappa, foi alcançado o grau de concordância intraexaminador de 0,92 e interexaminadores de 0,90.

Os dados foram analisados empregando-se técnicas de estatística descritiva e os resultados expressos em tabelas. A normalidade dos dados foi verificada por meio do teste de D'Agostino-Pearson. A associação entre o local de nascimento e a presença de cárie dentária foi analisada por meio do teste Qui-quadrado. A comparação das médias do índice CPOD e dos componentes dentes cariados, dentes perdidos e dentes obturados, entre os indivíduos nascidos e não nascidos no município onde o estudo foi conduzido, foi realizada por meio do teste de Mann-Whitney. O processamento e a análise dos dados foram realizados com auxílio do software EpiInfo versão 7.2.2, adotando-se um nível de significância de 5\%.

O estudo foi aprovado pelo Comitê de Ética em Pesquisa com Seres Humanos da Faculdade de Odontologia de Araçatuba - FOA-UNESP (CAAE $n^{\circ}$ 11036219.9.0000.5420). Atividades de educação em saúde voltadas à prevenção dos principais problemas de saúde bucal presentes na adolescência foram realizadas previamente à realização da pesquisa. Durante estas atividades, os escolares foram esclarecidos sobre o propósito e a metodologia do estudo e receberam o Termo de Assentimento Livre e Esclarecido (TALE) para que assinassem voluntariamente e, para aqueles que concordaram em participar do estudo, foi entregue o Termo de Consentimento Livre e Esclarecido (TCLE), para obtenção das autorizações de seus responsáveis legais. Também foi entregue questionário para obtenção de informações sobre o local de nascimento e crescimento do escolar.

\section{Resultados}

Observou-se que do total de 164 escolares, 51,83\% eram do sexo feminino, e 48,17\% do sexo masculino. Houve equilíbrio na composição dos grupos caso e controle em relação às variáveis sexo, nível de escolaridade do chefe da família e renda familiar mensal, como demonstrado na tabela 1 . O valor médio do índice CPOD, considerando todos os participantes, foi de $1,00 \pm 1,24$. 
Tabela 1. Perfil socioeconômico dos escolares de 12 anos de acordo com os grupos. Araçatuba, São Paulo, 2019.

\begin{tabular}{|c|c|c|c|c|c|c|}
\hline \multirow{2}{*}{ Variáveis } & \multicolumn{2}{|c|}{ Grupo caso } & \multicolumn{2}{|c|}{ Grupo controle } & \multicolumn{2}{|c|}{ Total } \\
\hline & $\mathrm{n}$ & $\%$ & $\mathrm{n}$ & $\%$ & $\mathrm{n}$ & $\%$ \\
\hline \multicolumn{7}{|l|}{ Sexo } \\
\hline Feminino & 42 & 51,22 & 43 & 52,44 & 85 & 51,83 \\
\hline Masculino & 40 & 48,78 & 39 & 47,56 & 79 & 48,17 \\
\hline Total & 82 & 100,00 & 82 & 100,00 & 164 & 100,00 \\
\hline \multicolumn{7}{|c|}{ Nível de escolaridade do chefe da família } \\
\hline Ensino fundamental incompleto & 42 & 51,22 & 44 & 53,66 & 86 & 52,44 \\
\hline Ensino fundamental completo & 10 & 12,20 & 9 & 10,98 & 19 & 11,59 \\
\hline Ensino médio incompleto & 13 & 15,85 & 12 & 14,63 & 25 & 15,24 \\
\hline Ensino médio completo & 11 & 13,41 & 13 & 15,85 & 24 & 14,63 \\
\hline Ensino superior incompleto & 2 & 2,44 & 1 & 1,22 & 3 & 1,83 \\
\hline Ensino superior completo & 4 & 4,88 & 3 & 3,66 & 7 & 4,27 \\
\hline Total & 82 & 100,00 & 82 & 3,66 & 164 & 100,00 \\
\hline \multicolumn{7}{|l|}{ Renda familiar mensal em reais } \\
\hline Até 500,00 & 9 & 10,98 & 9 & 10,98 & 18 & 10,98 \\
\hline Entre 500,00 e $1.500,00$ & 48 & 58,54 & 51 & 62,20 & 99 & 60,37 \\
\hline Entre $1.500,00$ e $2.500,00$ & 17 & 20,73 & 15 & 18,29 & 32 & 19,51 \\
\hline Mais que $2.500,00$ & 8 & 9,76 & 7 & 8,54 & 15 & 9,15 \\
\hline Total & 82 & 100,00 & 82 & 100,00 & 164 & 100,00 \\
\hline
\end{tabular}

Conforme visto na Tabela 2, a análise da relação entre o fator de exposição e a experiência de cárie dentária demonstrou que houve associação estatisticamente significante ( $\mathrm{p}$-valor=0,0010) entre não ter nascido e vivido sempre no município que realiza regularmente a fluoretação das águas de abastecimento público e apresentar índice CPOD>0. O odds ratio observado foi de 3,0134 (IC 95\%=1.5944 - 5.6953).

Verificou-se que os locais de nascimento dos escolares selecionados para compor a amostra eram, além do próprio município onde o estudo foi realizado, outras cidades do estado de São Paulo, Paraíba, Pernambuco, Piauí, Maranhão, Bahia, Paraná, Distrito Federal, e de outros países, sendo eles Venezuela, França e Japão.

Tabela 2. Relação entre a experiência de cárie dentária em escolares de 12 anos nascidos e não nascidos em localidade que realiza regularmente a fluoretação das águas de abastecimento público. Araçatuba, São Paulo, 2019.

\begin{tabular}{lrrrrrrrr}
\hline \multicolumn{1}{c}{ Fator de exposição } & \multicolumn{3}{c}{ Experiência de cárie dentária } & & \\
& \multicolumn{1}{c}{ CPOD>0 } & \multicolumn{2}{c}{ CPOD=0 } & \multicolumn{2}{c}{ Total } \\
& $\mathrm{n}$ & $\%$ & $\mathrm{n}$ & $\%$ & $\mathrm{n}$ & $\%$ \\
\hline Nasceram e/ou viveram em outras localidades & 54 & 65,85 & 32 & 39,02 & 86 & 52,44 \\
Nasceram e viveram sempre no município estudado & 28 & 34,15 & 50 & 60,98 & 78 & 47,56 \\
Total & 82 & 100,00 & 82 & 100,00 & 164 & 100,00 \\
\hline
\end{tabular}

Fonte: Autores.

Os escolares que nasceram e viveram sempre no município estudado apresentaram valores médios do índice CPOD $(\mathrm{p}<0,0001)$ e de seus componentes número de dentes cariados $(\mathrm{p}=0,0357)$ e número de dentes obturados $(\mathrm{p}=0,0049)$ estatisticamente menores em relação aos escolares que nasceram e/ou viveram em outras localidades (Tabela 3). Nenhum dente perdido devido à cárie dentária foi identificado durante os exames clínicos. 
Tabela 3. Média e desvio-padrão do índice CPOD e de seus componentes em escolares de 12 anos nascidos e não nascidos em localidade que realiza regularmente a fluoretação das águas de abastecimento público. Araçatuba, São Paulo, 2019.

\begin{tabular}{lcccc}
\hline \multicolumn{1}{c}{ Variáveis } & \multicolumn{2}{c}{ Local de nascimento } \\
& $\begin{array}{c}\text { Nasceram e viveram sempre no } \\
\text { município estudado }\end{array}$ & $\begin{array}{c}\text { Nasceram e/ou viveram } \\
\text { em outras localidades }\end{array}$ & p-valor \\
\hline Índice CPOD & $0,44 \pm 0,67$ & $1,31 \pm 1,32$ & $<0,0001$ \\
Componente dentes cariados & $0,12 \pm 0,34$ & $0,49 \pm 0,89$ & 0,0357 \\
Componente dentes obturados & $0,40 \pm 0,73$ & $0,83 \pm 1,05$ & 0,0049 \\
Componente dentes perdidos & 0 & 0 & - \\
\hline
\end{tabular}

Fonte: Autores.

\section{Discussão}

No presente estudo de caso-controle sobre a condição de saúde bucal de escolares de 12 anos matriculados em escolas da rede pública de ensino, foi comprovado que houve associação entre a menor experiência de cárie dentária e o fato de terem nascido e vivido sempre no município que fluoreta regularmente suas águas de abastecimento público. A severidade da doença também foi maior entre os escolares provenientes de outras localidades, que apresentaram maior número de dentes cariados e de dentes obturados, componentes do índice CPOD, indicando piores condições de saúde bucal.

Salienta-se que, considerando os exames de todos os participantes, o valor médio do índice CPOD encontrado neste estudo foi de 1,00, situando-se no intervalo de severidade da doença classificado pela Organização Mundial da Saúde como "muito baixo" (de 0 a 1,1) para indivíduos de 12 anos (World Health Organization, 2003). Ademais, os resultados encontrados por este estudo sugerem que população investigada apresenta melhores condições de saúde bucal em comparação com os dados de registros nacionais. De acordo com os resultados da mais recente Pesquisa Nacional de Saúde Bucal (SBBrasil 2010), realizada em todo o território brasileiro, o índice CPOD médio observado em jovens brasileiros de 12 anos de idade foi de 2,07, sendo verificadas variações dependendo da macrorregião do país, com a região norte apresentando o índice CPOD médio mais alto $(3,16)$ e a região sudeste o mais baixo $(1,72)$ (Brasil, 2012).

A manutenção da saúde bucal é fundamental para o crescimento e desenvolvimento adequado de crianças e adolescentes, com evidências de que problemas bucais podem gerar prejuízos na qualidade de vida dos indivíduos, afetando inclusive o bem-estar social e emocional (Ferreira et al., 2012). Nesse sentido, revisão sistemática recente constatou que crianças e adolescentes que possuem cárie dentária e pior condição de saúde bucal apresentam baixo desempenho escolar e menor frequência escolar (Rebelo et al., 2019). Assim, evidencia-se a importância de medidas de promoção e prevenção da saúde bucal para crianças e adolescentes, contribuindo para o desenvolvimento saudável e pleno da população.

A partir da análise do valor do odds ratio encontrado no presente estudo, verificou-se que os indivíduos de 12 anos que não nasceram e cresceram na localidade estudada tem uma chance 3,0134 vezes maior de ter cárie dentária em relação aos que nasceram e viveram sempre no município. Desta forma, é possível aventar que os indivíduos que tiveram acesso à água fluoretada regularmente durante toda a vida estiveram mais protegidos contra a cárie dentária. Ressalta-se que houve equilíbrio na proporção de indivíduos que compuseram os grupos caso e controle em relação ao sexo, nível de escolaridade do chefe da família e renda familiar mensal, sugerindo que os resultados não foram diretamente influenciados por essas variáveis.

Convém salientar que embora seja um método preventivo reconhecidamente eficaz e de grande abrangência, a fluoretação das águas de abastecimento público, realizada de modo a fornecer o máximo benefício preventivo contra a cárie dentária, com os teores de fluoreto dentro dos intervalos considerados ótimos e recomendados cientificamente, ainda não é alcançada em todas as localidades brasileiras. As principais dificuldades encontradas na implementação e manutenção da fluoretação das águas podem estar relacionadas à manutenção dos equipamentos necessários, recursos financeiros destinados à 
execução e monitoramento do método, capacitação de recursos humanos e complexidade da rede de distribuição de água (Garbin et al., 2017; Moimaz et al., 2015). Evidências demonstram que a dificuldade no controle da fluoretação das águas não se restringe aos municípios de pequeno porte, como demonstrado em um estudo que analisou o processo de vigilância dos níveis de flúor nas águas de abastecimento público nas capitais brasileiras, constatando que, na maior parte delas, os teores de flúor não eram monitorados corretamente (Cesa, Abegg, \& Aerts, 2011). Além disso, o Brasil apresenta informações insuficientes e desatualizadas sobre o potencial hídrico de seus aquíferos e a qualidade e características de suas fontes de águas, evidenciando a necessidade de maior compromisso intersetorial para se qualificar a fluoretação das águas no país, de forma que os teores cientificamente recomendados sejam alcançados e que toda a população tenha acesso a ela, principalmente as de maior vulnerabilidade social (Moimaz \& Santos, 2015). Nesse contexto, destaca-se que uma das principais vantagens da fluoretação das águas de abastecimento em relação a outros métodos preventivos para cárie dentária é que, além de apresentar grande abrangência, pode diminuir as desigualdades em saúde entre grupos de diferentes condições sociais, com custo operacional baixo (Moimaz et al., 2020)

As águas de abastecimento do município onde a pesquisa foi realizada são monitoradas por meio de um projeto de heterocontrole dos teores de fluoreto desde 2004, no qual amostras de água são coletadas diretamente da rede de distribuição em pontos preestabelecidos, abrangendo todas as fontes de captação de água da cidade, e analisadas mensalmente (Moimaz et al., 2018). Dessa forma, é possível sugerir que os escolares que nasceram e viveram sempre nesta localidade tiveram acesso à água de abastecimento público fluoretada nas concentrações recomendadas, proporcionando o máximo benefício na prevenção da cárie dentária. Salienta-se que, no período de 2004 a 2017, a média da concentração de flúor no município estudado foi de 0,69 $\mathrm{mgF} / \mathrm{L}$ e considerando que as médias das temperaturas máximas anuais na localidade se situam entre $26,3{ }^{\circ} \mathrm{C}$ e $32,5^{\circ} \mathrm{C}$, a concentração recomendada de flúor nas águas, que oferece o melhor benefício para prevenção da cárie dentária, varia de 0,55 a 0,84 mgF/L (Moimaz et al., 2018; Centro Colaborador do Ministério da Saúde em Vigilância da Saúde Bucal, 2011).

Os achados do presente estudo são corroborados por revisões sistemáticas que verificaram menor prevalência de cárie dentária em regiões com fluoretação das águas em comparação com regiões que não executam o método e evidenciam que a manutenção do acesso à água fluoretada nos teores recomendados desde o nascimento está associado a melhores condições de saúde bucal (McDonagh et al., 2000; Iheozor-Ejiofor et al., 2015). Isto está de acordo com o mecanismo de ação do flúor na cavidade bucal, visto que a exposição contínua ao flúor, em concentrações recomendadas, durante os periódicos processos de desmineralização e remineralização dentária promove a formação de reservas de fluoreto de cálcio, apatita fluoretada e fluorapatita na superfície dentária, proporcionando maior resistência à dissolução ácida resultante do metabolismo bacteriano (Rosin-Grget et al., 2013). Assim, reforça-se que para se atingir os efeitos preventivos desejados com o uso do flúor, sua presença na cavidade bucal deve ser mantida continuamente durante toda a vida do indivíduo (Garbin et al., 2017).

Em relação aos teores de flúor presentes nas águas de abastecimento das demais localidades de onde os escolares eram provenientes, embora esta variável tenha sido controlada, não há como comprovar que os escolares tiveram acesso à água com concentrações de flúor que proporcionem o máximo benefício preventivo, o que pode ser considerado uma limitação do estudo.

Convém destacar a importância da fluoretação das águas de abastecimento público como uma medida preventiva coletiva que contribui para promover a equidade em saúde. À medida que os métodos tópicos de utilização de flúor para prevenção da cárie dentária foram disseminados, principalmente por meio dos dentifrícios fluoretados, surgiram questionamentos sobre à real validade e necessidade da fluoretação das águas após a elucidação do mecanismo de ação tópica, entretanto, o método se faz necessário justamente por ser acessível à grande parte da população, principalmente para os indivíduos que poderiam ter menor capacidade de compra de produtos fluoretados ou dificuldade de acesso à aplicações de géis e soluções em consultórios (Garbin et al., 2017).

As populações de alto risco social estão mais susceptíveis à cárie dentária e suas sequelas, portanto, as ações de 
prevenção e promoção de saúde devem ser incentivadas por políticas públicas inclusivas e que garantam o acesso destes grupos. Sendo a utilização do flúor um dos principais responsáveis pela diminuição da prevalência de cárie dentária no mundo, a fluoretação das águas constitui-se no meio mais abrangente de seu uso.

\section{Conclusão}

A experiência de cárie dentária foi menor nos escolares que nasceram e viveram sempre no município com fluoretação das águas de abastecimento público do que nos escolares de outras localidades. Estudo futuros devem ser realizados visando aprofundar o conhecimento sobre a influência de hábitos alimentares e de variáveis socioeconômicas na ocorrência de cárie dentária nesta população.

\section{Agradecimentos}

O presente trabalho foi realizado com apoio da Coordenação de Aperfeiçoamento de Pessoal de Nível Superior - Brasil (CAPES) - Código de Financiamento 001.

\section{Referências}

Abanto, J., Tsakos, G., Paiva, S. M., Carvalho, T. S., Raggio, D. P., \& Bönecker, M. (2014). Impact of dental caries and trauma on quality of life among 5- to 6-year-old children: perceptions of parents and children. Community Dentistry and Oral Epidemiology, 42(5), 385-394. https://doi.org/10.1111/cdoe.12099

Araújo, P. C., Vettore, M. V., Baker, S. R., Garbin, C. A. S., Moimaz, S. A. S., \& Arcieri, R. M. (2018). Oral health knowledge among Brazilian preschoolers and their carers receiving and not receiving healthpromotion. Bioscience Journal, 34(2), 514-524. https://doi.org/10.14393/BJ-v34n2a2018-39508

Baghlaf, K., Muirhead, V., Moynihan, P., Weston-Price, S., \& Pine, C. (2018). Free sugars consumption around bedtime and dental caries in children: a systematic review. JDR Clinical and Translational Research, 3(2), 118-129. https://doi.org/10.1177/2380084417749215

Brasil. (2012). SB Brasil 2010: Pesquisa Nacional de Saúde Bucal: resultados principais. Ministério da Saúde.

Carolino, R. A., Silva, G. G., Silva, M. E. C., Silva, T. A. F., Silva, I. P. A., Paiva, D. F. F., Xerez, M. C., Pinheiro, J. C., Silva, R. U. O., Medeiros, R. F. S. B., Souza, L. C., \& Moura, C. (2020). Evaluation of the impact of oral health conditions on the quality of life of schoolchildren: An exploratory study. Research, Society and Development, 9(10), e2849108683. https://doi.org/10.33448/rsd-v9i10.8683

Centro Colaborador do Ministério da Saúde em Vigilância da Saúde Bucal. (2011). Consenso técnico sobre classificação de águas de abastecimento público segundo o teor de flúor. São Paulo: Faculdade de Saúde Pública da Universidade de São Paulo.

Cesa, K., Abegg, C., \& Aerts, D. (2011). A vigilância da fluoretação de águas nas capitais brasileiras. Epidemiologia e Serviços de Saúde, $20,547-555$.

Dye, B. A., Hsu, K. L., \& Afful, J. (2015). Prevalence and measurement of dental caries in young children. Pediatric Dentistry, 37(3), 200-216.

Ferreira, M. C., Goursand, D., Bendo, C. B., Ramos-Jorge, M. L., Pordeus, I. A., \& Paiva, S. M. (2012). Agreement between adolescents' and their mothers' reports of oral health-related quality of life. Brazilian Oral Research, 26(2), 112-118. http://dx.doi.org/10.1590/S1806-83242012000200005

Frazão, P. (2012). Epidemiology of dental caries: when structure and context matter. Brazilian Oral Research, 26(spe1), 108-114. https://doi.org/10.1590/S180683242012000700016

Garbin, C. A. S., Santos, L. F. P., Garbin, A. J. I., Moimaz, S. A. S., \& Saliba, O. (2017). Fluoridation of public water supply: bioethical, legal and political approach. Revista Bioética, 25(2), 328-337. https://doi.org/10.1590/1983-80422017252193

Gomes, D., \& Ros, M. A. D. The etiology of carie: the construction of a thought-style. (2008). Ciência \& Saúde Coletiva, 13(3), 1081-1090. https://doi.org/10.1590/S1413-81232008000300031

Hall-Scullin, E., Whitehead, H., Milsom, K., Tickle, M., Su, T. L., \& Walsh, T. (2017). Longitudinal study of caries development from childhood to adolescence. Journal of Dental Research, 96(7), 762-767. https://doi.org/10.1177/0022034517696457

Iheozor-Ejiofor, Z., Worthington, H. V., Walsh, T., O'Malley, L., Clarkson, J. E., Macey, R., Alam, R., Tugwell, P., Welch, V., \& Glenny, A. M. (2015). Water fluoridation for the prevention of dental caries. The Cochrane Database of Systematic Reviews, $2015(6)$, CD010856. https://doi.org/10.1002/14651858.CD010856.pub2

Instituto Brasileiro de Geografia e Estatística. (2021). Cidades e estados: Araçatuba. https://www.ibge.gov.br/cidades-e-estados/sp/aracatuba.html

Malta, M., Cardoso, L. O., Bastos, F. I., Magnanini, M. M., \& Silva, C. M. F. (2010). STROBE initiative: guidelines on reporting observational studies. Revista de Saúde Pública, 44(3), 559-565. https://doi.org/10.1590/S0034-89102010000300021 
Research, Society and Development, v. 10, n. 6, e9910615646, 2021

(CC BY 4.0) | ISSN 2525-3409 | DOI: http://dx.doi.org/10.33448/rsd-v10i6.15646

McDonagh, M. S., Whiting, P. F., Wilson, P. M., Sutton, A. J., Chestnutt, I., Cooper, J., Misso, K., Bradley, M., Treasure, E., \& Kleijnen, J. (2000). Systematic review of water fluoridation. British Medical Journal, 321(7265), 855-859. https://doi.org/10.1136/bmj.321.7265.855

Moimaz, S. A. S., \& Santos, L. F. P. (2015). Estudo longitudinal da fluoretação das águas em município com complexa rede de distribuição: dez anos de estudo. Archives of Health Investigation, 4(5), 11-16.

Moimaz, S. A. S., Garbin, C. A. S., Iglesias, G. F., Chiba, F. Y., Sumida, D. H., \& Saliba, O. (2015). Dificuldades enfrentadas no processo de fluoretação das águas de abastecimento público. Revista Brasileira de Pesquisa em Saúde, 17(1), 87-94.

Moimaz, S. A. S., Santos, L. F. P., Saliba, N. A., \& Saliba, O. (2017). Eleven years of monitoring fluoride content in public water supplies: methods, products, and importance to public health. Bioscience Journal, 33(3), 799-808. https://doi.org/10.14393/BJ-v33n3-36328

Moimaz, S. A. S., Santos, L. F. P., Saliba, T. A., Saliba, N. A., \& Saliba, O. (2020). Health surveillance: public water supply fluoridation in 40 municipalities of São Paulo, Brazil. Ciência \& Saúde Coletiva, 25(7), 2653-2662. https://doi.org/10.1590/1413-81232020257.03972018

Moimaz, S. A. S., Santos, L. F. P., Saliba, T. A., Saliba, N. A., \& Saliba, O. (2018). Heterocontrole do flúor nas águas de abastecimento público: resultados e experiência de 13 anos de vigilância. Archives of Health Investigation, 7(7), 262-268. https://doi.org/10.21270/archi.v7i7.3037

Mota, K. R., Oliveira, A. C. N., Heimer, M. V., Moreira, A. R. O., Pugliesi, D. M. C., \& Santos Junior, V. E. (2020). Public oral health policies for children: historical perspective, state of the art and future challenges. Research, Society and Development, 9(11), e3529119869. https://doi.org/10.33448/rsd-v9i11.9869

Rebelo, M. A., Vieira, J. M. R., Pereira, J. V., Quadros, L. N., \& Vettore, M. V. (2019). Does oral health influence school performance and school attendance? A systematic review and meta-analysis. International Journal of Paediatric Dentistry, 29(2), 138-48. https://doi.org/10.1111/ipd.12441

Righolt, A. J., Jevdjevic, M., Marcenes, W., \& Listl, S. (2018). Global-, regional-, and country-level economic impacts of dental diseases in 2015. Journal of Dental Research, 97(5), 501-507. https://doi.org/10.1177/0022034517750572

Rosin-Grget, K., Peros, K., Sutej, I., \& Bašić, K. (2013). The cariostatic mechanisms of fluoride. Acta Medica Academica, 42(2), 179-188. http://dx.doi.org/10.5644/ama2006-124.85

Silva, M. H. A., Fernandes, E. C., Monteiro, A. K. A. P., \& Santos, P. B. D. (2020). Knowledge of parents, teachers and dentists about fluoride toothpaste in children under 7 years. Research, Society and Development, 9(8), e78985351. http://dx.doi.org/10.33448/rsd-v9i8.5351

Souza, J. G. S., Souza, S. E., Noronha, M. S., Ferreira, E. F., \& Martins, A. M. E. B. L. (2017). Impact of untreated dental caries on the daily activities of children. Journal of Public Health Dentistry, 78(3), 197-202. https://doi.org/10.1111/jphd.12259

World Health Organization. (2003). The World Oral Health Report 2003. Continuous improvement of oral health in the 21st century: the approach of the WHO Global Oral Health Programme. Geneva: WHO.

World Health Organization. (2013). Oral health surveys: basic methods. (5a ed.), WHO.

World Health Organization. (2015). Prevention is better than treatment. Bulletin of the World Health Organization, 93, 594-595. http://dx.doi.org/10.2471/BLT.15.020915 